\title{
Application of contrast-enhanced ultrasound in the diagnosis of burn depth
}

\author{
Xue Jiang ${ }^{1}$, Feng $\mathrm{Li}^{2}$, Yunfei Chi ${ }^{2}$, Xin Chen ${ }^{2}$, Yukun Luo ${ }^{3}$, Qinggui Ye ${ }^{1}$, Wenjing Song ${ }^{1}$, Guoping Li $^{4}$ \\ ${ }^{1}$ Department of Ultrasound Diagnosis, The Fourth Medical Center of PLA General Hospital, Beijing, China; ${ }^{2}$ Burns and Plastic Department, The \\ Fourth Medical Center of PLA General Hospital, Beijing, China; ${ }^{3}$ Department of Ultrasound Diagnosis, The First Medical Center of PLA General \\ Hospital, Beijing, China; ${ }^{4}$ Grand Life Science \& Technology, Ltd., Beijing, China \\ Contributions: (I) Conception and design: F Li; (II) Administrative support: F Li; (III) Provision of study materials and animals: F Li, Y Chi, X Chen; \\ (IV) Collection and assembly of data: X Jiang, Q Ye, W Song; (V) Data analysis and interpretation: X Jiang, Y Luo; (VI) Manuscript writing: All \\ authors; (VII) Final approval of manuscript: All authors. \\ Correspondence to: Feng Li, MD. Burns and Plastic Department, The Fourth Medical Center of PLA General Hospital, Beijing, China. \\ Email: lifeng3041@163.com.
}

Background: The diagnosis of burn depth often relies on the subjective judgment of plastic surgeons. Contrast-enhanced ultrasound (CEUS) can visualize the microcirculation well and has potential value in diagnosing the depth of burn wounds. We assessed the depth of the burn wounds by CEUS, and compared the results with histological examination.

Methods: Two rhesus monkeys were used, and multiple burn wounds with different depths were made on their backs. The echo of the dermis and subcutaneous tissue were observed for each wound, and the thickness of the dermis was measured. CEUS was performed to evaluate the depth of burn wounds and compared with pathological results.

Results: (I) After scalding, dermal tissue edema occurred, and the thickness of the dermis measured by a US tended to increase gradually, related to the time of scalding and the order of measurement. (II) With the prolongation of the burn time, the depth of filling by contrast agent gradually increased, from the superficial dermis to the deep dermis and subcutaneous tissue, indicating that the depth of tissue damage gradually increased. This was consistent with the pathological observation. The thickness of the healthy dermis was about $1.3-1.8 \mathrm{~mm}$, and $2.7-4.1 \mathrm{~mm}$ after scalding. The depth of the burn wounds was $0.9-4.1 \mathrm{~mm}$, accounting for $32-100 \%$ of the full skin thickness.

Conclusions: CEUS is a convenient and fast examination method that is consistent with pathological diagnosis of the depth of burn wounds and could prove valuable for the accurate assessment of burn injuries.

Keywords: Cutaneous burns; contrast-enhanced ultrasound (CEUS); animal study; pathological diagnosis

Submitted Jun 09, 2021. Accepted for publication Aug 11, 2021.

doi: $10.21037 / \mathrm{atm}-21-3715$

View this article at: https://dx.doi.org/10.21037/atm-21-3715

\section{Introduction}

Accurate diagnosis of burn depth has great significance for evaluating burn injuries. To date has mainly relied on the plastic surgeon's clinical experience and subjective judgment, leading to misjudgment of the injury, delays to treatment, or over-treatment. Studies have shown that the accuracy of plastic surgeons' subjective judgment of burn depth is only about $60-70 \%$ (1).

Imaging examinations can provide objective evidence for the diagnosis of many diseases. Among the various imaging modalities, ultrasound (US), a combination of gray-scale and color Doppler flowing imaging (CDFI), is widely used because of its convenience and real-time observations. A US with a high-frequency transducer can observe the structure of superficial tissues in gray-scale, including skin $(2,3)$, 
and it has been used to assess skin and subcutaneous tissue inflammation, tumors, and skin condition (4-6). CDFI can be used to observe blood flow; however, sensitivity is low in detecting tissue blood vessels, and it is difficult to detect low-speed blood flow signals. The dermal blood vessels are mainly composed of a network of capillaries, so color Doppler US alone cannot accurately observing the signals of capillaries in the dermis. Contrast-enhanced US (CEUS) is widely used in clinical practice. The contrast agent increases the signal and contrast of tissues (7), and thus, tiny blood vessels with a diameter of 10-30 $\mu \mathrm{m}$ and a flow rate of about $1 \mathrm{~mm} / \mathrm{s}$ can be observed (8). CEUS clearly shows the microcirculation of tissues and has been used in the burns field, mainly to judge tissue viability after skin flap transplantation $(9,10)$. However, there is no related research on the use of CEUS to evaluate burn depth. We assumed that CEUS with a high-frequency transducer might better assess skin thickness and blood flow perfusion. In the current study, we utilized animal models of different burn depths to explore the efficacy of CEUS in diagnosing the depth of burn wounds in comparison with pathological examination as verification. We present the following article in accordance with the ARRIVE reporting checklist (available at https://dx.doi.org/10.21037/atm-21-3715).

\section{Methods}

The experiment was conducted under the project license (No. 20210627) granted by Beijing xierxin experimental animal ethics committee, in compliance with the National Institutes of Health Guide for the care and use of animals and Regulations for the Administration of Affairs Concerning Ex-Perimental Animals promulgated by the State Science and Technology Commission. A protocol was prepared before the study without registration.

\section{Animal model}

A male and female adult healthy rhesus monkey, weighing 9 and $6.4 \mathrm{~kg}$ respectively, were used before the experiment, single cage adaptive feeding for 1 week. The animal feeding room is a fully enclosed indoor cage made of iron mesh angle iron, which is equipped with heating, lighting, habitat rack, water dispenser, etc. The fan is used to extract air. In winter, the indoor temperature is $17-22^{\circ} \mathrm{C}$, and the lighting adopts natural daylighting. The dorsum was shaved using clippers. Anesthesia was induced with compound ketamine solution, diluted with 1:9 with normal saline, and injected intramuscularly at $0.1-0.15 \mathrm{~mL} / \mathrm{kg}$ of body weight. The animals were fixed on the animal operating table in a prone position, and saphenous vein access was established. Burn injury was created with a digital temperature control scald instrument developed by the Fourth Medical Center of PLA General Hospital. It had four parts: a digital temperature control system, a scald head, a round copper scald module, and a temperature calibration system. The temperature error could be controlled within $\pm 1.0^{\circ} \mathrm{C}$. The temperature of the copper scald head was adjusted to $110^{\circ} \mathrm{C}$, preheated for $15 \mathrm{~min}$, and then held close to different parts of the back of the monkey and for 5, 8, 10, 12 or 15 s to form 2 $\mathrm{cm}$ diameter wounds with different depths, separated by a certain distance. The normal skin area at a certain distance next to the scald focus was used as the control $(0 \mathrm{~s})$. The animals were not killed after the experiment. It can be used in other types of experiments after wound healing.

\section{Ultrasound evaluation}

We used the M9 portable US system (Mindray, China), linear array probe L12-4s, probe frequency 4-12 MHz, and SonoVue contrast agent (Bracco, Italy). SonoVue is phospholipid-encapsulated sulfur hexafluoride microbubbles. Each US observation of the scald wounds was adjusted to the same parameters. The mechanical index (MI) was set to 0.086 . Immediately after the scald wounds have been made, US examinations were performed. A solid gel pad was used to cover the wound to keep the skin in the focusing area of the acoustic beam to prevent degradation of the US image.

The wounds were firstly observed under gray-scale conditions. The echo of the dermis and subcutaneous tissue were observed, and the thickness of the dermis was measured. Next, CDFI was performed to observe the blood perfusion of the dermis and subcutaneous tissue.

For imaging under contrast conditions, the US instrument was adjusted to dual-frame contrast imaging for dynamic observation of the gray-scale and contrast images simultaneously. The contrast agent was dissolved with $5 \mathrm{~mL}$ normal saline and mixed well; $1.0 \mathrm{~mL}$ of the contrast medium suspension was injected as an intravenous bolus and then rinsed with $5.0 \mathrm{~mL}$ of normal saline for each wound evaluation. The timer was turned on, and for $120 \mathrm{~s}$ each burn wound was observed to fill the contrast agent in the dermis. When the contrast agent had subsided completely, the next wound was then observed. The ultrasound doctor who performed this examination was blinded to the burn 


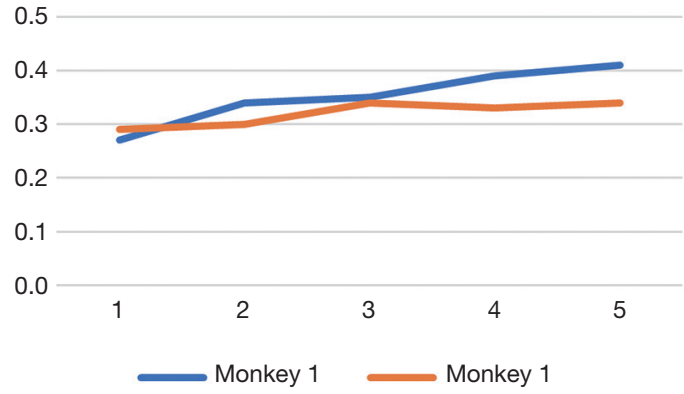

Figure 1 Results of dermal thickness measurements in two animals. The abscissa is the order of the scalding wound examined, and the ordinate is the thickness of the dermis $(\mathrm{cm})$. The animals were measured in reversed order, yet both showed a tendency of thickening of the dermis after scalding.

depth. The doctor had more than 10 years of experience in US as well as contrast-enhanced US examinations.

\section{Image analysis}

For all the wounds created by different scald times, the echo, boundary, and shape of the US images and the blood perfusion status of the skin were observed under CDFI conditions. The measurement order for the first animal started with the wound burned for $5 \mathrm{~s}$, and then $8 \mathrm{~s}, 10 \mathrm{~s}$, $12 \mathrm{~s}$, and finally $15 \mathrm{~s}$. The measurement order for the second animal was the reverse from the wound burned for $15 \mathrm{~s}$, and then $12 \mathrm{~s}, 10 \mathrm{~s}, 8 \mathrm{~s}$, and finally $5 \mathrm{~s}$. In the CEUS images, the contrast agent enhancement mode in the arterial phase and the depth of the contrast agent filling were measured.

\section{Pathological examination}

Tissue biopsies of the center of the wounds were harvested with a 5 -mm full-thickness punch, including epidermis, dermis, and subcutaneous tissue, which were then fixed in $10 \%$ formalin. Routine dehydration, clearing, wax immersion, paraffin embedding, and 4- $\mu \mathrm{m}$ sectioning were performed, and sections were stained with hematoxylin and eosin.

\section{Results}

\section{General observation}

Within a short period after the scald, the appearance of the wounds' surface for each burn duration was roughly similar.
The size and shape of the wounds were basically the same as the copper scald head, and the boundary between the wounds and normal skin was clear. The wounds were white, and their surfaces were flush with the surrounding normal skin in the early post-injury period, then gradually swelled, without redness or blisters.

\section{US image analysis}

Under the gray-scale US, the epidermal line-like strong echo disappeared in the scald wounds, becoming rough and uneven, showing low echo with uneven thickness, and scattered high echoes in places. The dermis showed varying degrees of thickening. For the first monkey, the dermal thickness of the wounds was measured in ascending order of burn time, and for the second monkey, measured in the reverse order. With the delay in examination time, the dermis thickness increased in both animals (Figure 1). In the dermis, there was no significant difference in the internal echoes among the wounds. They showed fusiform or elliptical hypoechoic areas, the boundary was not clear, and the internal echo was relatively uniform, slightly lower than the surrounding normal dermal echo. Under the condition of CDFI, no obvious blood flow signal was found in any of the wounds.

Following the injection of the contrast agent, the edge of the wound burned for $5 \mathrm{~s}$ was completely enhanced, and only about one-third of the superficial dermis was not enhanced. Enhancement was observed at the edge of the wound, and about half the depth of the dermis in the wound burned for $8 \mathrm{~s}$. Enhancement was not observed at the edge of the wound burned for $10 \mathrm{~s}$, and was observed in about the lower one-third of the dermis. In the wound burned for $12 \mathrm{~s}$, enhancement was observed at the deep edge of the dermis, and the complete boundary between the dermis and the subcutaneous tissue could be clearly seen. In the wound burned for $15 \mathrm{~s}$, the deep boundary of the dermis was enhanced, but part of the boundary between the dermis and the subcutaneous was defective (Figure 2). The results for skin thickness and wound depth measured by US are shown in Table 1.

\section{Histological observations}

In the wound burned for $5 \mathrm{~s}$, the whole layer of the epidermis was denatured and necrotic, one-third of the superficial dermis was coagulated and necrotic, and degeneration of the fibrous matrix was observed; however, 

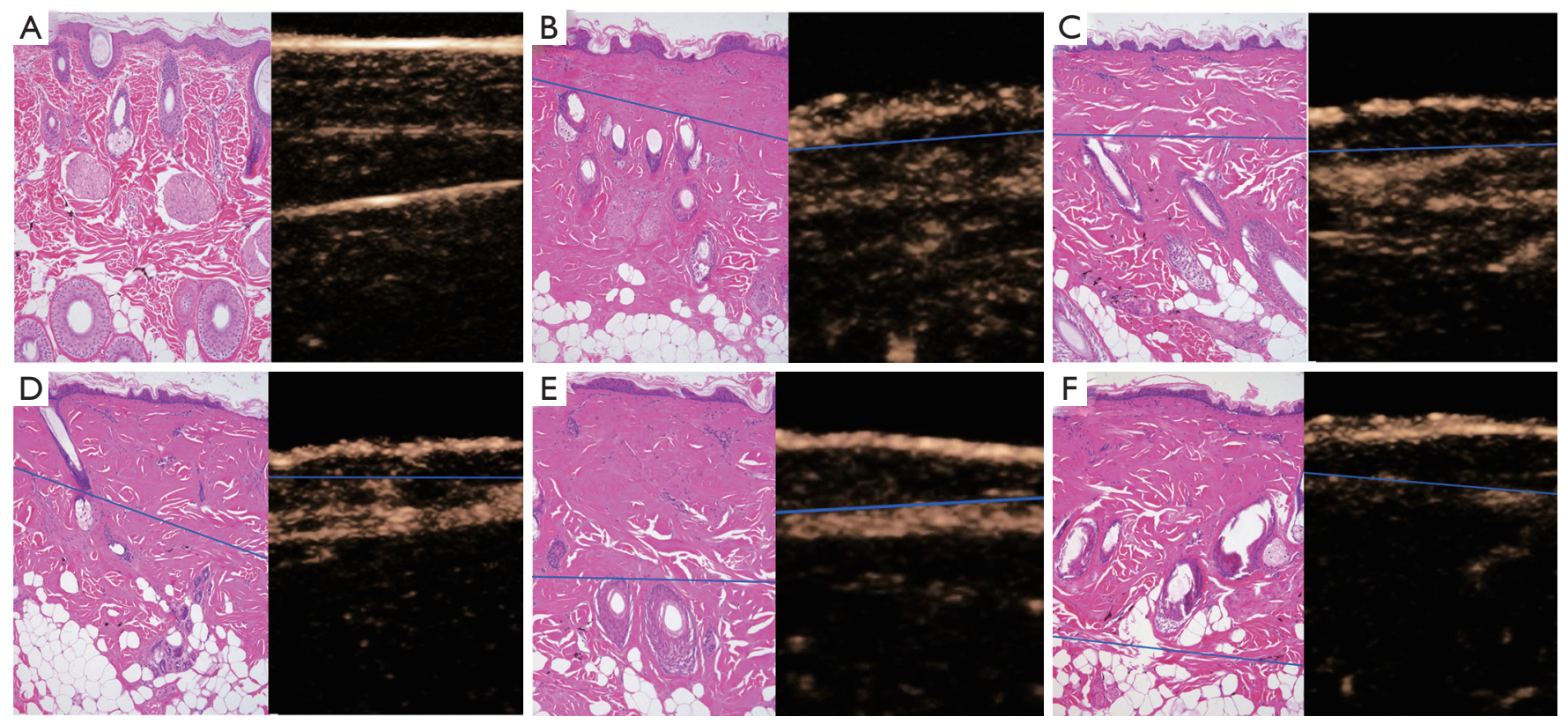

Figure 2 Comparison of HE-stained $(\times 200)$ histological slides and contrast-enhanced ultrasound (US) images. The blue line in the histological slides indicates the boundary between the denatured necrotic tissue and healthy tissue. The blue line in the contrast-enhanced US (CEUS) image indicates the boundary between the areas with and without blood perfusion. (A) Normal dermis and subcutaneous tissue. CEUS shows full-thickness enhancement of the dermis. (B) The 5-s scalding site, showing the depth of the wound is the superficial layer of the dermis. (C) The 8-s scalding site, with the depth of the wound reaching the middle of the dermis. (D) The 10-s scalding site, with the depth of the wound in about the lower one-third of the dermis. (E) The 12-s scalding site, showing that the wound extends to the basal layer of the dermis. (F) The 15-s scalding site, showing the depth of the wound between the dermis and the subcutaneous tissue. The US images were highly consistent with the histological results. Scale bar: $100 \mu \mathrm{m}$.

Table 1 Skin thickness (mm) and wound depth ( $\mathrm{mm})$ measured by ultrasound

\begin{tabular}{|c|c|c|c|c|c|c|}
\hline Inspection method & \multicolumn{6}{|c|}{ Duration of scald } \\
\hline \multicolumn{7}{|l|}{ Monkey 1} \\
\hline Gray-scale & 1.3 & 2.7 & 3.4 & 3.5 & 3.9 & 4.1 \\
\hline CEUS & 0 & 0.9 & 1.8 & 2.1 & 3.5 & 4.1 \\
\hline \multicolumn{7}{|l|}{ Monkey 2} \\
\hline Gray-scale & 1.8 & 3.4 & 3.3 & 3.4 & 3.0 & 2.9 \\
\hline CEUS & 0 & 1.1 & 1.5 & 2.3 & 2.5 & 2.9 \\
\hline CEUS/gray-scale (\%) & 0 & 32.4 & 45.5 & 67.6 & 83.3 & 100 \\
\hline
\end{tabular}

CEUS, contrast-enhanced ultrasound.

the structure of the underlying tissue was roughly normal. In the wound burned for $8 \mathrm{~s}$, more than half of the dermal tissue was coagulated and necrotic, there was intercellular fibrous degeneration, but deep hair follicles and sebaceous glands were roughly intact. In the wound burned for $10 \mathrm{~s}$, about two-thirds of the dermis and most of the matrix 
fibers degenerated, the dermis was destroyed, the nucleus deformed, and the chromatin tended to be uniform. The underlying fibrous matrix was generally normal. Most structures of the dermis appeared degenerated and necrotic in the wound burned for $12 \mathrm{~s}$. In the wound burned for $15 \mathrm{~s}$, part of the subcutaneous fat structure below the dermis was damaged, and the hair follicle cell layer was blurred, and the skin appendages were destroyed completely (Figure 2).

\section{Discussion}

The diagnosis of burn depth is not an unresolved problem, but in clinical practice, the judgment of burn depth often relies on the subjective experience of plastic surgeons. Therefore, different judgments may be made about the same wound, which will inevitably affect the prognostic judgment and the adoption of appropriate treatments. It is difficult to obtain objective data to assess wound depth, which is the main reason for inaccurate diagnosis. How to avoid the subjectivity of diagnosis is a long-standing unresolved problem in the field of burns injury. Histological examination has long been regarded as the gold standard, but it is difficult to carry out routinely as an invasive examination.

Many studies have tried to find non-invasive and equivalent alternatives. Stasis or occlusion of the microcirculation is an important feature of seriously burned tissue. Some researchers have found that the degree of burn depth is closely related to the state of tissue microcirculation, so the depth of burns can be assessed by the microcirculation of the wound (11-14), such as with laser Doppler imaging (LDI), laser speckle-imaging (LSI) and spatial frequency domain imaging (SFDI). LDI combines laser imaging and Doppler blood flow imaging to capture the Doppler frequency shift of red blood cells moving relative to the laser source, so the microcirculation of the tissue can be observed. The technical principle of LSI is similar to that of LDI, but the tissue microcirculation can be observed in a shorter time (15-17). SFDI is an imaging method based on diffuse reflection, which can provide tissue blood flow information and even discover collagen denaturation. However, LDI measurement takes a long time, and when SFDI inspects the curved skin surface, measurement errors will occur $(18,19)$. In addition, these inspection methods can only observe the microperfusion of the tissue and cannot accurately measure the thickness of the dermis. Therefore, blood perfusion measurement alone cannot accurately determine the depth of dermal injury, so these methods are of limited help in judging the severity of burns.

High-frequency US can observe the structure of the epidermis, dermis, and subcutaneous tissue and measure each layer, and US contrast imaging can observe the microcirculation of the tissue. Therefore, we used CEUS with a high-frequency transducer to accurately diagnose burn depth, which was verified by pathological examination.

In the present study, because the permeability of the capillaries increases and serum leaks out, edema of the burn wounds was observed firstly. We measured the thickness of the wound dermis in the order from 5 to $15 \mathrm{~s}$ scald in the first animal, while that for the second animal was in the reverse order. According to our measurements, the thickness of the healthy dermis was about $1.3-1.8 \mathrm{~mm}$, which increased to $2.7-4.1 \mathrm{~mm}$ after scald. Due to edema appearing immediately after scald, the degree of skin thickness increase was related to the time of scald and the order of measurement.

Based on the degree of tissue edema, some studies have reported that the use of terahertz imaging could determine the depth of burns (20-22). However, terahertz imaging cannot observe the tissue structure and the state of the microcirculation, so solely relying on the degree of edema to assess burn depth has obvious limitations.

We found that the results from high-frequency CEUS were highly consistent with the pathological examination in the diagnosis of burn wound depth in the present study. Under US contrast conditions, with prolongation of scald time, it could be clearly seen that the depth of microcirculation ischemia deepened from the superficial dermis to the deep dermis, and the boundary of the contrast medium filling gradually migrated to the deep part of the tissue. When the burn lasted for $15 \mathrm{~s}$, the full dermis and part of the subcutaneous tissue were damaged; no blood perfusion could be observed in the whole dermis or the upper subcutaneous tissue, which reflected a full-thickness burn. The evolution process was basically consistent with the pathological observations. The depth of the burn wounds could be measured accurately by combining contrast-enhanced US with the gray-scale US. In the present work, burn depth ranged from 0.9 to $4.1 \mathrm{~mm}$, accounting for $32-100 \%$ of the full skin thickness. The ratio of burn depth to full skin thickness increased gradually with the extension of burn time. This continuous and dynamic process proved the accuracy of this imaging method in diagnosing burn depth.

In conclusion, the present study is the first attempt to 
use the combination of high-frequency US and CEUS to reflect burn depth through assessment of the tissue microcirculation, which was consistent with the pathological examinations. It could provide an objective imaging basis for diagnosing the depth of deep partial-thickness burns because compared with superficial partial-thickness burns and full-thickness burns, deep partial-thickness burns are a broader concept, and the severity of this kind of injury is not easy to distinguish based on clinical experience. As a convenient technique, the method we proposed in this study may have good prospects for application in burn wound depth diagnosis but has yet to be tested in further clinical practice.

The advantage of this study is that the damage of dermal microcirculation can be observed by contrast-enhanced ultrasound under high-frequency ultrasound; Observe the structure of the skin and subcutaneous tissue and accurately measure the thickness of the dermis and subcutaneous tissue. The limitations of this method include: This method is suitable for measuring the wound depth of a point but not for measuring the wound depth of a surface.

\section{Acknowledgments}

Funding: Beijing Municipal Commission of Science and Technology (Z181100001718037).

\section{Footnote}

Reporting Checklist: The authors have completed the ARRIVE reporting checklist. Available at https://dx.doi. org/10.21037/atm-21-3715

Data Sharing Statement: Available at https://dx.doi. org/10.21037/atm-21-3715

Conflicts of Interest: All authors have completed the ICMJE uniform disclosure form (available at https://dx.doi. org/10.21037/atm-21-3715). Dr. GL is from Grand Life Science \& Technology, Ltd. The other authors have no conflicts of interest to declare.

Ethical Statement: The authors are accountable for all aspects of the work in ensuring that questions related to the accuracy or integrity of any part of the work are appropriately investigated and resolved. The experiment was conducted under the project license (No. 20210627) granted by Beijing xierxin experimental animal ethics committee, in compliance with the National Institutes of Health Guide for the care and use of animals and Regulations for the Administration of Affairs Concerning Ex-Perimental Animals promulgated by the State Science and Technology Commission.

Open Access Statement: This is an Open Access article distributed in accordance with the Creative Commons Attribution-NonCommercial-NoDerivs 4.0 International License (CC BY-NC-ND 4.0), which permits the noncommercial replication and distribution of the article with the strict proviso that no changes or edits are made and the original work is properly cited (including links to both the formal publication through the relevant DOI and the license). See: https://creativecommons.org/licenses/by-nc-nd/4.0/.

\section{References}

1. Mirdell R, Farnebo S, Sjöberg F, et al. Interobserver reliability of laser speckle contrast imaging in the assessment of burns. Burns 2019;45:1325-35.

2. O'Rourke K, Kibbee N, Stubbs A. Ultrasound for the Evaluation of Skin and Soft Tissue Infections. Mo Med 2015;112:202-5.

3. Schuetzenberger K, Pfister M, Messner A, et al. Comparison of optical coherence tomography and high frequency ultrasound imaging in mice for the assessment of skin morphology and intradermal volumes. Sci Rep 2019;9:13643.

4. Nelson CE, Chen AE, Bellah RD, et al. Ultrasound features of purulent skin and soft tissue infection without abscess. Emerg Radiol 2018;25:505-11.

5. Waller JM, Maibach HI. Age and skin structure and function, a quantitative approach (I): blood flow, $\mathrm{pH}$, thickness, and ultrasound echogenicity. Skin Res Technol 2005;11:221-35.

6. Crişan D, Badea AF, Crişan M, et al. Integrative analysis of cutaneous skin tumours using ultrasonogaphic criteria. Preliminary results. Med Ultrason 2014;16:285-90.

7. Miele V, Piccolo CL, Galluzzo M, et al. Contrastenhanced ultrasound (CEUS) in blunt abdominal trauma. Br J Radiol 2016;89:20150823.

8. Zhang L, Gu J, Zhao Y, et al. The role of multimodal ultrasonic flow imaging in Thyroid Imaging Reporting and Data System (TI-RADS) 4 nodules. Gland Surg 2020;9:1469-77.

9. Geis S, Prantl L, Gehmert S, et al. TTP (time to PEAK) and RBV (regional blood volume) as valuable parameters 
to detect early flap failure. Clin Hemorheol Microcirc 2011;48:81-94.

10. Lamby P, Prantl L, Fellner C, et al. Post-operative monitoring of tissue transfers: advantages using contrast enhanced ultrasound (CEUS) and contrast enhanced MRI (ceMRI) with dynamic perfusion analysis? Clin Hemorheol Microcirc 2011;48:105-17.

11. Crouzet C, Nguyen JQ, Ponticorvo A, et al. Acute discrimination between superficial-partial and deep-partial thickness burns in a preclinical model with laser speckle imaging. Burns 2015;41:1058-63.

12. Karim AS, Shaum K, Gibson ALF. Indeterminate-Depth Burn Injury-Exploring the Uncertainty. J Surg Res 2020;245:183-97.

13. Wearn C, Lee KC, Hardwicke J, et al. Prospective comparative evaluation study of Laser Doppler Imaging and thermal imaging in the assessment of burn depth. Burns 2018;44:124-33.

14. Watts AM, Tyler MP, Perry ME, et al. Burn depth and its histological measurement. Burns 2001;27:154-60.

15. Wang XQ, Mill J, Kravchuk O, et al. Ultrasound assessed thickness of burn scars in association with laser Doppler imaging determined depth of burns in paediatric patients. Burns 2010;36:1254-62.

16. Ponticorvo A, Rowland R, Baldado M, et al. Evaluating clinical observation versus Spatial Frequency Domain Imaging (SFDI), Laser Speckle Imaging (LSI) and

Cite this article as: Jiang X, Li F, Chi Y, Chen X, Luo Y, Ye Q, Song W, Li G. Application of contrast-enhanced ultrasound in the diagnosis of burn depth. Ann Transl Med 2021;9(16):1315. doi: $10.21037 / \mathrm{atm}-21-3715$ thermal imaging for the assessment of burn depth. Burns 2019;45:450-60.

17. Wang R, Zhao J, Zhang Z, et al. Diagnostic Accuracy of Laser Doppler Imaging for the Assessment of Burn Depth: A Meta-analysis and Systematic Review. J Burn Care Res 2020;41:619-25.

18. Ponticorvo A, Burmeister DM, Yang B, et al. Quantitative assessment of graded burn wounds in a porcine model using spatial frequency domain imaging (SFDI) and laser speckle imaging (LSI). Biomed Opt Express 2014;5:3467-81.

19. Burmeister DM, Ponticorvo A, Yang B, et al. Utility of spatial frequency domain imaging (SFDI) and laser speckle imaging (LSI) to non-invasively diagnose burn depth in a porcine model. Burns 2015;41:1242-52.

20. Dutta M, Bhalla AS, Guo R. THz Imaging of Skin Burn: Seeing the Unseen-An Overview. Adv Wound Care (New Rochelle) 2016;5:338-48.

21. Taylor ZD, Singh RS, Culjat MO, et al. Reflective terahertz imaging of porcine skin burns. Opt Lett 2008;33:1258-60.

22. Tewari P, Garritano J, Bajwa N, et al. Methods for registering and calibrating in vivo terahertz images of cutaneous burn wounds. Biomed Opt Express 2019;10:322-37.

(English Language Editor: K. Brown) 